\title{
Fish oil supplementation reduces maternal defensive inflammation and predicts a gut bacteriome with reduced immune priming capacity in infants
}

\author{
Candice Quin ${ }^{1} \cdot$ Deanna M. Vollman ${ }^{1} \cdot$ Sanjoy Ghosh ${ }^{1} \cdot$ Natasha Haskey $\mathbb{D}^{1} \cdot$ Mehrbod Estaki $^{1} \cdot$ Jason Pither $^{1}$. \\ Jacqueline A. Barnett ${ }^{1} \cdot$ Michael N. Jay ${ }^{1,2} \cdot$ Blake W. Birnie $^{1,2} \cdot$ Deanna L. Gibson $^{1,2}$
}

Received: 11 July 2019 / Revised: 24 April 2020 / Accepted: 29 April 2020 / Published online: 12 May 2020

(c) The Author(s), under exclusive licence to International Society for Microbial Ecology 2020

\begin{abstract}
Habitual supplementation of fish oil is thought to provide benefits to the developing infant; however, the effects on infant microbial establishment and immune development are unknown. A 6-month observational cohort study was conducted where 47 out of 91 women self-administered dietary fish oil during breastfeeding. Infant stool and mothers' breast milk were collected each month over 6 months. Gas chromatography was used to quantify breast milk fatty acids and high-throughput sequencing was used to assess the infant fecal microbiota. Immune markers and parent-reported questionnaires were used to assess infant immunity and health up to 2 years. Our results reveal that fish oil supplementation decreased secretory immunoglobulin A and increased IL-10 production in lactating women along with increased breast milk eicosapentaenoic acid, and this corresponded to increased abundances of fecal Bifidobacterium and Lactobacillus spp. in their infants. Docosahexaenoic acid levels in breast milk aligned with decreases in infant gut bacterial richness and the predicted bacterial phenotypes suggested that fish oil lowers commensal traits involved in pathogen colonization resistance. Despite this, there were no differences in sickness incidence in toddlers. This study revealed that fish oil associates with decreases in breast milk defensive inflammatory responses and corresponds with infant fecal microbiota with anti-inflammatory potential.
\end{abstract}

\section{Introduction}

Prenatal supplementation with fish oil, rich in n-3 polyunsaturated fatty acids (PUFA) like eicosapentaenoic acid (EPA, C20:5n-3) and docosahexaenoic acid (DHA, C22:6n3 ), is recommended since deficiency negatively impacts visual development [1], behavior [2], and cognition [3]. Accordingly, n-3 PUFAs are predicted to provide beneficial effects on infant health and development [4]. The neonatal period is a critical time for development of the intestinal

Supplementary information The online version of this article (https:// doi.org/10.1038/s41396-020-0672-9) contains supplementary material, which is available to authorized users.

Deanna L. Gibson

deanna.gibson@ubc.ca

1 Department of Biology Okanagan Campus, University of British Columbia, Kelowna, BC, Canada

2 Department of Medicine, Faculty of Medicine, University of British Columbia, Kelowna, BC, Canada microbiome and the immune system, both of which influence enteric infection susceptibility. We have previously shown that the gut microbiome alters enteric infection in rodents [5]. Of the factors important in shaping the gut microbiome in neonates, breast milk is the most influential [6]. While fish oil supplementation during lactation has been hypothesized to be beneficial on infant development [7], three recent meta-analyses concluded that n-3 PUFAs do not improve physiological outcomes [8-10] and may suppress infant immunity [10].

Dietary n-3 PUFAs decrease pro-inflammatory responses [11-15], and supplementation does increase risk for infection (reviewed in [16]). Similarly, we have shown that mice fed fish oil have an altered microbiome and immune cell balance which correlated with fatal sepsis during acute colitis infection [17, 18]. Other rodent studies also found dietary n-3 PUFA increased susceptibility to enteric pathogens like Helicobacter pylori [19], Mycobacterium tuberculosis [20, 21], Listeria monocytogenes [22, 23], Salmonella enterica Enteritidis [24], as well as respiratory pathogens like Paracoccidioides brasiliensis [25] and influenza [26-28]. While the former studies were 
postnatally exposed to fish oil, we have shown that maternal fish oil exposure in rodents resulted in their offspring preprogrammed in their T-cell biology [29], shown to increase susceptibility to colitis [30]. If these findings hold true in humans, the widespread practice of prenatal fish oil supplementation could provoke dysfunctional gut immunity in infants. It is currently unknown how fish oil supplementation affects intestinal colonization by the microbiota during infancy, a critical time when immune tolerance and disease susceptibility patterns develop.

The purpose of this study was to determine if fish oil supplementation during breastfeeding alters infant immunity and gut microbiota. We found that fish oil increased EPA in breast milk corresponding with a decrease in secretory immunoglobulin A (sIgA). These breast milk alterations were associated with an infant microbial composition that predicted reduced immune priming, although there were no changes in self-reported illness between the two groups over a 2-year period. These data suggest that fish oil supplmentation to lactating mothers could be a risk factor for increasing susceptibility to infectious disease in their offspring.

\section{Subjects and methods}

\section{Subjects and study design}

The research protocol was approved by the UBC Clinical Research Ethics Board and BC Interior Health Ethics Board, and written informed consent was obtained from participants. The trial was conducted according to Declaration of Helsinki principles and registered at https:// clinicaltrials.gov (identifier NCT03297801). Study design, recruitment, and subjects are previously described [31]. Briefly, a prospective cohort clinical study was conducted in the Okanagan Valley, BC, Canada where lactating women, who either did or did not supplement their diets with fish oil until their infants were 6 months of age, were recruited. The inclusion criteria required medically certified healthy, fullterm infants who obtained over $75 \%$ of their daily nutritional intake from breast milk. Preterm infants $(\leq 37$ weeks of gestation), infants diagnosed with congenital disorders, or infants introduced to solid foods were excluded. There were no exclusion criteria for the mothers.

One hundred and nine women-infant pairs were recruited, where 18 were excluded and the remaining 91 women (plus one twinset) were included in the final analysis. Fortyseven women self-administered fish oil (on average $500 \pm$ $104 \mathrm{mg}$ EPA and $303 \pm 63 \mathrm{mg}$ DHA daily) over the 6month period and 44 women did not supplement. Information about delivery, antibiotic treatment, anthropometric measurements of the infant, formula intake, vaccination compliance, and demographics of the parents was collected monthly to account for confounding variables. Of the participants included, 7 infants received formula in the hospital and 14 (8 in fish oil and 6 in no fish oil cohort) were partially supplemented with formula milk over the course of the study.

\section{Infant stool and breast milk sample collection}

Infant stool samples were collected, stored, and processed as previously described [32], at 1 week of age, and once per month until 6 months. Concurrently, breast milk samples from the mothers were collected the morning of infant stool collection. Before sample collection, wearing sterile gloves, the women cleaned the nipple and surrounding area with warm water and soap, and manually expressed a few drops of milk before collecting $10 \mathrm{~mL}$ of foremilk in a sterile tube. The part of the pump that contacted the breast and all the materials used to collect milk were cleansed well and sterilized before each use. All samples were kept at $-20{ }^{\circ} \mathrm{C}$ for $<4 \mathrm{~h}$ before being transported to the laboratory using dry ice, and then stored at $-80^{\circ} \mathrm{C}$ until analysis. Instructions and sterile collection packages for samples were given to the participants every month.

\section{Breast milk fatty acids and dietary intake analysis}

To understand the relationship between dietary intake and breast milk fatty acid composition, participants were asked to fill out a 24-h dietary recall each sample collection day to correlate dietary intake and supplementing patterns with the coinciding stool and breast milk samples collected. The dietary questionnaires were assessed by a research dietitian and entered into the NCI automated self-administered (ASA24) dietary assessment tool, a web-based tool for 24-h dietary analysis. The data output from this tool included a summary of dietary nutrients in grams, consumed by the participants. Grams of dietary lipids were converted to relative abundances for direct comparison with the lipids quantified from breast milk. Since dietary recalls are not always good indicators of breast milk fatty acids, the medium- and long-chain fatty acids in breast milk were then chemically quantified using gas chromatography (GC) as previously described [33]. To understand if the two cohorts (mothers self-administering fish oil supplements or not) had different breast milk fatty acids while accounting for infant age, a multivariate analysis of variance (MANOVA) was used. Of the chemically quantified breast milk fatty acids, C13:0, C16:0, C18:1n-9, C18:2n-6, C20:1n-9, C20:5n-3 (EPA), C22:5n-3, and C22:6n-3 (DHA) were selected as explanatory variables based on a priori interest and published animal models. To evaluate the relationship between dietary recalls and quantified breast milk lipids, a pairwise 
rank correlation followed by a Procrustes rotation and PROTEST permutation test was performed.

\section{Immune function markers}

Immune markers, $\operatorname{sg} \mathrm{A}, \operatorname{IgE}$, and pro and anti-inflammatory cytokines [granulocyte-macrophage colony-stimulating factor, interferon gamma (IFN- $\gamma$ ), interleukin (IL)- $1 \beta$, IL2, IL-6, IL-8, IL-10, IL-12 (p70), monocyte chemoattractant protein-1, tumor necrosis factor alpha (TNF- $\alpha$ ), IL-13, and IL-5] were measured from breast milk and stool supernatant at 1 week, 1 month and 5 months of age, using multiplex assays (Eve Technologies Calgary, Canada).

\section{Sickness incidence}

Infant mucosal illness was recorded by primary caregivers for a 2-year time period using an online sickness incidence form as described [31]. Caregivers were asked to complete the online questionnaire each time their infants were sick and were sent monthly reminders.

\section{$16 \mathrm{~S}$ sequencing methods}

16S rRNA sequencing was used for bacterial identification [34] of the V3-V4 region, as previously [31]. The $2 \times 300$ paired end reads were sequenced on a MiSeq system with a V3 chemistry kit at The Applied Genomics Core in Edmonton, $\mathrm{AB}$.

\section{Bioinformatics}

All bioinformatics was conducted using Quantitative Insights into Microbial Ecology (QIIME2) [35]. Forward sequences underwent quality filtering, dereplication, chimera removal and denoising using the Deblur plugin [36] with default settings. A Naive Bayes classifier was trained for our primer sets using the Greenegenes (13_8) reference database sequences based on $99 \%$ similarity. Taxonomic classification was collapsed and assigned at each phylogenetic level. For phylogenetic-based analyses, MAFFTaligned [37] sequences were used to produce a phylogeny tree using the fast-tree method [38] with default settings. Microbial data were rarefied to a sampling depth of 4380 for the whole cohort and 5924 for exploratory subset analysis and had an average frequency of 19,738 and 19,330 per sample, respectively.

\section{Statistical methods}

Statistical analyses were performed using $\mathrm{R}$ [39] version 3.3.0. Results are expressed as mean values \pm standard errors of the mean (SEM) unless otherwise stated. For demographic and dietary fish consumption comparisons, Fisher's exact and chi-squared tests were used. A Pearson correlation analysis assessed associations between dietary intake and breast milk fatty acids. Phyloseq was used to generate alpha diversity indexes and distance matrices based on the rarefied feature table. A pairwise comparison of alpha diversity measures was conducted in QIIME2 followed by a mixed-effects model using lme4 to account for infant sex, mode of delivery (MOD), probiotic supplementation, and infant age. Microbial communities in the fish oil groups were visualized using a PCoA on a Bray-Curtis dissimilarity matrix the Vegan package and visualized using ggplot2. LEfSe evaluated statistical significance and effect size of taxa abundances [40] where features with fewer than ten counts across all samples or appearing in fewer than five samples were removed. A multiclass comparison was performed using the Kruskal-Wallis test $(\alpha=0.05)$, and a linear discriminant analysis (LDA) score for discriminative features set to 2.0. The biologically relevant features were visualized in a cladogram. BugBase [41] determined if fish oil associated with any predictive phenotypes and the type of bacteria was predicted using VSEARCH [42] at 97\% similarity. Statistical analysis of the predictions was done using a pairwise Mann-Whitney-Wilcoxon test.

\section{Relationship between breast milk fatty acids and the infant bacteriome}

A multiple regression analysis was conducted using an open source R code [43]. Only participants with complete data set including GC-quantified breast milk and infant stool samples at months 2,3 , and 4 were assessed resulting in 24 subjects for this exploratory analysis. All the included infants in this subset analysis were exclusively breast fed. Potential predictors of microbial composition were screened using a Spearman correlation matrix and fatty acids that exhibited a significant correlation with alpha diversity species richness were included. Multicollinearity between fatty acids was checked using the variable inflation factor index with a maximum cut-off score of 10 . The microbial communities were analyzed using two complementary multivariate approaches: (1) constrained ordination using RDA, focusing on community composition differences in relation to predictors of interest, and (2) multiple generalized linear models. Because of our interest in age effects, each infant age was assessed separately while acknowledging increased potential for type-1 errors. Fatty acids included: tridecylic acid (C13:0), palmitic acid (C16:0), oleic acid (C18:1n-9), linoleic acid (C18:2n-6), EPA (C20:5n-3), docosapentaenoic acid (C22:5n-3), and DHA (C22:6n-3). Abundance data at each taxonomic level along with the fatty acids were Hellinger transformed [44]. 
Variable selection in RDA was implemented using the ordistep function in Vegan version 2.4-4, using both forward and backward stepwise inclusion. Features with a significant correlation coefficient based on the first two axes (evaluated at a Bonferroni-adjusted alpha level) were displayed on the RDA plots with type II scaling. To evaluate the association of genus abundance with explanatory variables, we implemented multiple negative binomial GLMs using the mvabund package [45] version 3.12.3, which uses a series of univariate $F$-tests of the effects of predictor variables on the abundance of each taxon, while adjusting for multiple testing. Residual diagnostics were used to assess regression assumptions. Microbes were filtered and any taxa that made up $<0.1 \%$ of the total count and occurring in $<75 \%$ of samples were removed (cf. [46]). Seven taxa met the inclusion criteria at 3 months of age and four taxa met the inclusion criteria at 4 months of age and were included in the model. To explore the relationship between the microbial communities and the breast milk fatty acids over time and to test the significance of the identified relationship, Procrustes rotation and PROTEST permutation tests were implemented in Vegan based on the RDAs which showed a significant accord between breast milk fatty acids and infant stool microbial communities [47, 48]. A LDA using the MASS [49] package in $\mathrm{R}$ was used to determine whether the quantified immune markers could discriminate between the two supplementing groups. The collected data sets were first split into a training set and a test set. We trained the LDA model and then validated our results using the test data set to predict the supplementing group. This was followed by an MANOVA with Wilks' lambda test for comparisons. Immune data were first "scaled" in $\mathrm{R}$ using default settings to accommodate differences in measurements. IFN $\gamma$, IL-6, IL-8, IL-10, IL-12, TNF $\alpha$, IL-5, and SIgA were included in the LDA model and MANOVA at 1 and 5 months. Given sample size limitations, at 1 week of age the immune model was reduced to IL-10 and $\operatorname{sg}$ A, based on findings at 1 and 5 months. The remaining immune cells were tested using Student's $t$ tests. Non-detects were treated as zero values. For group comparisons of breast milk lipids, an MANOVA with Wilks' lambda test was ran on "logit" transformed lipid proportions at each age. In all cases, model fit was visually inspected using diagnostic plots of the residuals.

\section{Results}

The study aims were to: understand how habitual fish oil supplementation during lactation alters bioactive constituents in breast milk and infant stool; determine if fish oil supplementation was associated with changes to the infant microbial composition; explore the relationship between specific breast milk fatty acids and infant microbial diversity; and determine if fish oil supplementation corresponded with altered parent-reported incidence of infection by 2 years of age. The flow of participants throughout the trial is detailed in Fig. S1. Of the 91 mothers to complete the study, 62 provided at least one breast milk sample over a 6-month period for paired comparisons. Participants were mostly well-educated and of Euro-Canadian descent with similar household income, maternal age, gestational age, and infant birth characteristics between the two groups (Table S1).

\section{Fish oil supplementation associates with increased $\mathrm{n}-3$ PUFA in breast milk and decreased defensive inflammation}

No differences in dietary fish consumption were reported by the participants. The Procrustes rotation analyses and protest tests showed a significant accord between mothers reported dietary intake and fatty acids quantified in breast milk (Table 1). This suggested that the overall profile of lipid intake data of mothers from the questionnaire correlated with the overall profile of lipids measured in their breast milk. Without adjusting for multiple testing, pairwise rank correlations revealed significant associations between maternal dietary consumption and breast milk fatty acids, but the results were inconsistent over time (Table 1). Therefore, all forthcoming analyses using breast milk fatty acids were conducted on the GC-quantified fatty acids.

Assessment of the breast milk lipid profile (Fig. 1a) revealed that participants supplementing with fish oil (500 $\pm 104 \mathrm{mg}$ EPA and $303 \pm 63 \mathrm{mg}$ DHA daily average) had a higher proportion of EPA (C20:5n-3) in their breast milk at 1,4 , and 5 months postpartum despite no detectable differences in DHA (C22:6n-3) or alpha-linolenic acid (C18:3n-3) (Table S2). The mothers supplementing habitually with fish oil had lower amounts arachidonic acid (C20:4n-6) at 1 week and 2 months of breastfeeding as well as eicosatrienoic acid (C20:3n-3) at 1 week, 1, 3 and, 4 months of breastfeeding. Therefore, self-administration of fish oil supplements correlated with changes to the bioactive constituents in breast milk. The percent compositions of all quantified breast milk fatty acids are available in Table S2.

In addition to changes in fatty acid percent composition, changes to breast milk immune markers were associated with fish oil supplementation (Table S3). LDA predicted the supplementing group at 1 month with a $61.5 \%$ accuracy $(P=0.05)$ based on IFN- $\gamma$, IL-5, IL-6, IL-8, IL-10, IL-12, TNF- $\alpha$, and $\operatorname{sgA}$ concentrations in breast milk. The groups were different predominantly due to the higher antiinflammatory cytokine, IL-10 $(P=0.058)$ in the fish oil group. At 5 months, the LDA model predicted the supplementing group with a $62.5 \%$ accuracy $(P=0.05)$ between the two cohorts. Results from the MANOVA showed that 
Table 1 Comparison of reported maternal nutrient intake $24 \mathrm{~h}$ prior to breast milk collection and GC quantified breast milk fatty acids.

\begin{tabular}{|c|c|c|c|c|c|c|c|c|c|c|c|c|}
\hline \multirow[t]{2}{*}{ Dietary nutrient } & \multicolumn{2}{|l|}{2 months } & \multirow[t]{2}{*}{$R$} & \multirow[t]{2}{*}{$P$} & \multicolumn{2}{|l|}{3 months } & \multirow[t]{2}{*}{$R$} & \multirow[t]{2}{*}{$P$} & \multicolumn{2}{|l|}{4 months } & \multirow[t]{2}{*}{$R$} & \multirow[t]{2}{*}{$P$} \\
\hline & Recall & Breast milk & & & Recall & Breast milk & & & Recall & Breast milk & & \\
\hline Total FA & 100 & 100 & & & 100 & 100 & & & 100 & 100 & & \\
\hline SFA & $38.0 \pm 2.3$ & $42.5 \pm 1.6$ & 0.51 & $*$ & $37.1 \pm 2.0$ & $41.1 \pm 1.5$ & 0.58 & $*$ & $38.6 \pm 2.0$ & $41.6 \pm 1.3$ & 0.49 & $*$ \\
\hline C16:0 & $19.6 \pm 0.9$ & $19.5 \pm 0.6$ & 0.27 & & $19.1 \pm 0.8$ & $18.5 \pm 0.6$ & 0.5 & $*$ & $20.1 \pm 0.8$ & $17.7 \pm 0.6$ & 0.56 & $*$ \\
\hline MUFA & $39.1 \pm 1.2$ & $38.2 \pm 1.0$ & 0.62 & $*$ & $39.8 \pm 1.4$ & $39.3 \pm 1.1$ & 0.54 & $*$ & $40.6 \pm 1.2$ & $39.1 \pm 0.8$ & 0.3 & \\
\hline C18:1n-9 & $35.7 \pm 1.1$ & $33.8 \pm 0.9$ & 0.69 & $*$ & $36.0 \pm 1.6$ & $35.5 \pm 1.0$ & 0.47 & $*$ & $37.7 \pm 1.2$ & $35.0 \pm 0.8$ & 0.33 & \\
\hline PUFA & $22.9 \pm 1.7$ & $19.3 \pm 0.9$ & 0.26 & & $23.1 \pm 1.5$ & $19.6 \pm 0.9$ & 0.23 & & $20.9 \pm 1.5$ & $19.3 \pm 0.8$ & 0.5 & $*$ \\
\hline$C 18: 2 n-6$ & $19.5 \pm 1.6$ & $16.5 \pm 0.8$ & 0.39 & & $19.0 \pm 1.2$ & $16.8 \pm 0.8$ & 0.25 & & $17.9 \pm 1.3$ & $16.4 \pm 0.7$ & 0.48 & $*$ \\
\hline$C 20: 5 n-3$ & $0.2 \pm 0.1$ & $0.08 \pm 0.01$ & 0.31 & & $0.3 \pm 0.1$ & $0.1 \pm 0.02$ & 0.46 & $*$ & $0.1 \pm 0.0$ & $0.09 \pm 0.01$ & 0.13 & \\
\hline$C 22: 5 n-3$ & $0.02 \pm 0.0$ & $0.09 \pm 0.01$ & -0.2 & & $0.05 \pm 0.0$ & $0.1 \pm 0.02$ & 0 & & $0.02 \pm 0.0$ & $0.07 \pm 0.01$ & -0.2 & \\
\hline$C 22: 6 n-3$ & $0.2 \pm 0.1$ & $0.1 \pm 0.01$ & 0.45 & $*$ & $0.3 \pm 0.1$ & $0.1 \pm 0.02$ & 0.07 & & $0.2 \pm 0.0$ & $0.10 \pm 0.01$ & 0.17 & \\
\hline \multirow[t]{2}{*}{ Procrustes } & $P$ & $m_{12}$ & & & $P$ & $m_{12}$ & & & $P$ & $m_{12}$ & & \\
\hline & 0.015 & 0.78 & & & 0.011 & 0.77 & & & 0.01 & 0.77 & & \\
\hline
\end{tabular}

Breast milk fatty acids are expressed as a relative percent of all the medium and long-chain fatty acids measured. ASA dietary recall values are expressed as relative abundance of total grams of fatty acid consumed. Data are presented as mean $\pm \mathrm{SEM}$, $* P<0.05$ by MANOVA with Wilks' lambda test. Procrustes rotation analysis and PROTEST test $P$ values and correlation diagnostic $\left(m_{12}\right.$ which is equivalent to $\left.r^{2}\right)$ displayed. Values include only participants with complete breast milk and stool samples at 2, 3, and 4 months of age $(n=24)$.

*P $(<0.05)$ indicates a significant association between the dietary recall and the individual breast milk lipids as assessed by a Pairwise rank correlation at each age, separately.

the fish oil group had lower sIgA, an important mucosal defense factor (Fig. 1b). In contrast, IgE, which plays a role in allergies [50], was similar between the two groups. IL-10 and $\operatorname{sIg} A$ were included in the LDA model at 1 week and they predicted the fish oil group with a $75 \%$ accuracy $(P=$ 0.16). Overall, the LDA was able to discriminate between the two groups based on quantified immune markers where fish oil supplementation was associated with decreased protective sIgA and increased anti-inflammatory IL-10.

\section{Habitual fish oil supplementation augments bacteria with reduced immune priming potential}

Fecal bacterial diversity was assessed to determine if fish oil supplementation associated with changes to the infant microbial composition. The mixed-effects model found no overall difference in alpha diversity measures between the supplemented and non-supplemented cohorts (Fig. 2a); however, pairwise comparisons found lower Shannon's diversity and Pielou's evenness, two alpha diversity indexes, at 5 months in the fish oil group (Table S4). Pairwise comparisons of weighted and unweighted UniFrac distance matrices revealed marginal $(P=0.08)$ differences in the presence of fecal bacteria between the two infant groups at 1 week of age only. This observation was followed-up with LEfSe [40], which showed infants in the fish oil group had higher abundances of Bifidobacterium, Lactobacillus, and Bacteroides spp. in stool, whereas the non-fish oil group had an abundance of Firmicutes phyla (Fig. 2b). Collectively, these data show a positive association between fish oil supplementation and bacteria commonly present in high abundances in breast-fed and vaginally birthed babies that have been shown to induce anti-inflammatory responses [51, 52].

\section{Breast milk EPA and DHA predict infant gut microbial diversity}

\section{Breast milk DHA corresponds with lower alpha diversity measures in infants}

To gain a better understanding of which specific breast milk fatty acids influence the infant fecal microbiota, eight breast milk fatty acids of a priori interest were included as potential predictors of alpha diversity on corresponding infant stool sets at months 2, 3, and 4. In addition, infant sex and MOD were included as they have previously been shown to influence microbial diversity. We constructed separate multiple regressions for each diversity index, which exhibited collinearity (Figs. S2-S4). After prescreening, all the potential predictors using a Spearman correlation analysis, DHA (C22:6n-3) in breast milk, infant sex, and MOD were included in the multiple regression model (Table 2). At 2 months of age, species richness was negatively associated with breast milk DHA and vaginal deliveries (Fig. 3a). Faith's PD similarly showed a negative association with DHA but not infant sex or MOD. None of the predictors were associated with Shannon diversity or Pielou's Evenness at 2 months of age. At 3 months, vaginal deliveries negatively associated with Shannon diversity, but 

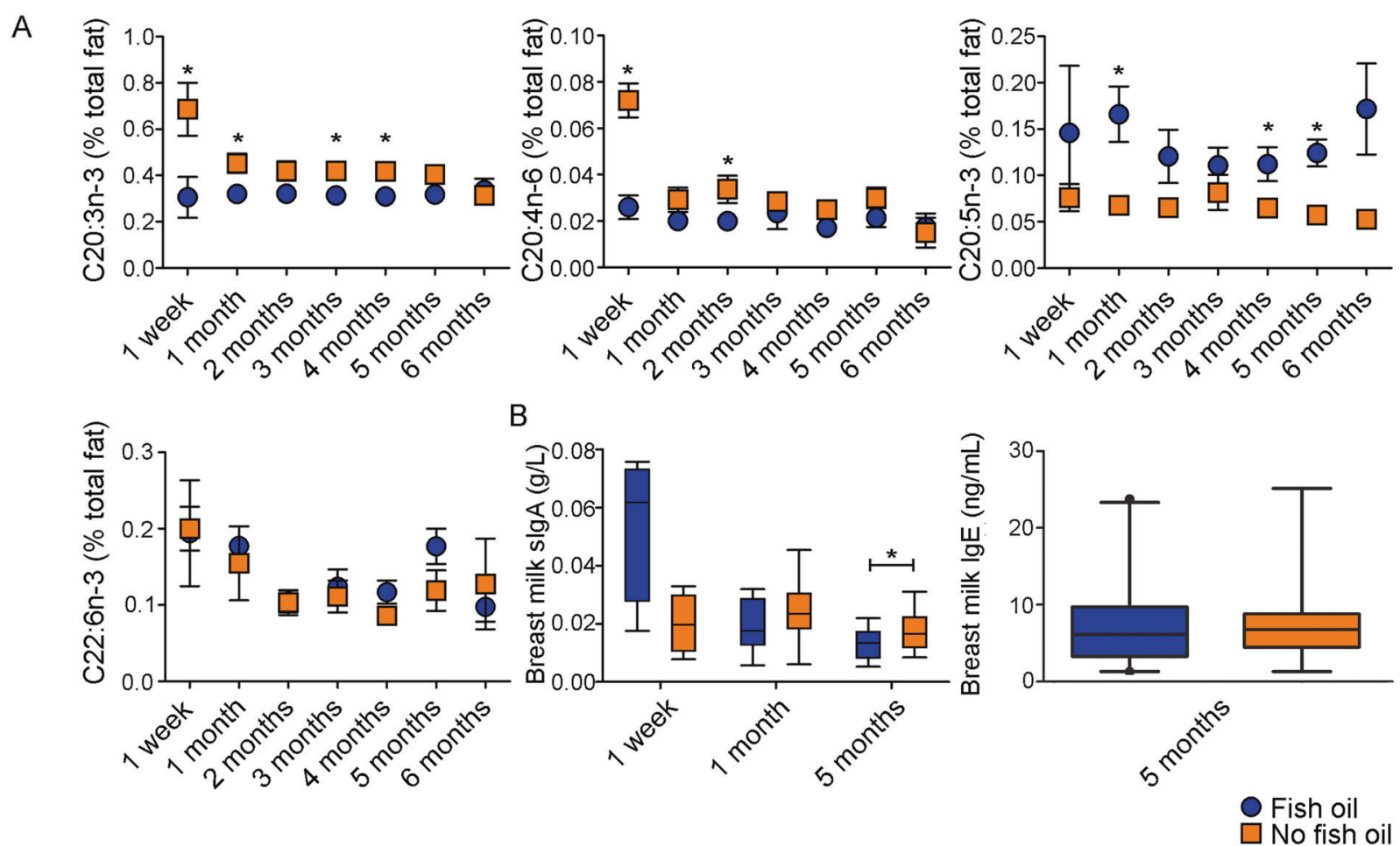

Fig. 1 Fish oil supplements taken during lactation alter the lipid profile in the breast milk and impair defensive immunity. a Compared with the non-fish oil group, breast milk of participants self-administering fish oil supplements during lactation had a significantly lower proportion of eicosatrienoic acid (C20:3n-3) and arachidonic acid (C20:4n-6) in their breast milk and a higher proportion of eicosapentaenoic acid (EPA, C20:5n-3), an omega-3 PUFA present in fish oil ( $n=$ fish oil: no fish oil at 1 week (5:5), 1 month
(15:13), 2 months (20:19), 3 months (23:19), 4 months (28:22), 5 months (28:19), 6 months (12:4)). There were no differences in docosahexaenoic acid (DHA, C22:6n-3) after correcting for multiple testing. $\mathbf{b}$ The prevalence of breast milk sIgA was similar between the two cohorts at 1 week and 1 month of age. In contrast, at 5 months the no fish oil group had higher breast milk sIgA (22:18). IgE was not affected by fish oil exposure at 5 months. $* P \leq 0.05$ by MANOVA. not species richness, Pielou's evenness, or Faith's PD (Fig. 3b). In contrast, Faith's PD positively correlated with male infants and the percent composition of breast milk palmitic acid (C16:0). At 4 months, DHA was negatively associated with species richness, Faith's PD, and Shannon diversity. Male sex was likewise positively associated with species richness and Faith's PD. MOD did not reach statistical significance (Fig. 3c). Overall, breast milk DHA corresponds with lower alpha diversity measures over time in the developing microbiome of infants.

\section{Overall gut bacterial community composition is dictated by EPA and DHA}

Overall, 84 genera from nine phyla were represented across all participants (Table 3). The fish oil group included representation from $57.7 \pm 3.1$ genera, while the no fish oil group was made up of $58.0 \pm 1.5$ genera at the reported ages. None of the breast milk fatty acids were selected as meaningful explanatory variables of infant fecal genera at 2 months of age, but oleic acid (C18:1n-9), EPA (C20:5n3), DHA (C22:6n-3), and MOD were selected at 3 months of age, and tridecylic acid (C13:0), EPA, DHA, and MOD were selected at 4 months of age as determined by Monte Carlo Permutation Procedure at 1000 permutations (Table S5). In all, $14.52 \%$ and $13.34 \%$ of the total variation in taxon composition was attributed to these fatty acids as explanatory variables at ages 3 and 4 months, respectively (Fig. 4). Genera that were significantly correlated with at least one of the first two RDA axes are highlighted. The results illustrate that EPA in breast milk correlated with Bifidobacterium, and Streptococcus spp. and MOD correlated with Bacteroides. While DHA was selected as a significant predictor at both months 3 and 4 , it did not consistently align with any of the selected genera. At 3 months DHA aligned with Enterobacteriaceae (Fig. 4a) but this finding was not apparent at 4 months (Fig. 4b). Oleic acid correlated with Lactobacillus spp. at 3 months and tridecylic acid with Bifidobacterium and Streptococcus spp. at 4 months, similar to EPA. Clostridium spp. correlated with RDA1 but was not associated with the predictor fatty acids included in our model. As EPA was increased in the breast milk of supplementing mothers, and this was validated using LEfSe. As expected, LEfSe shows an 

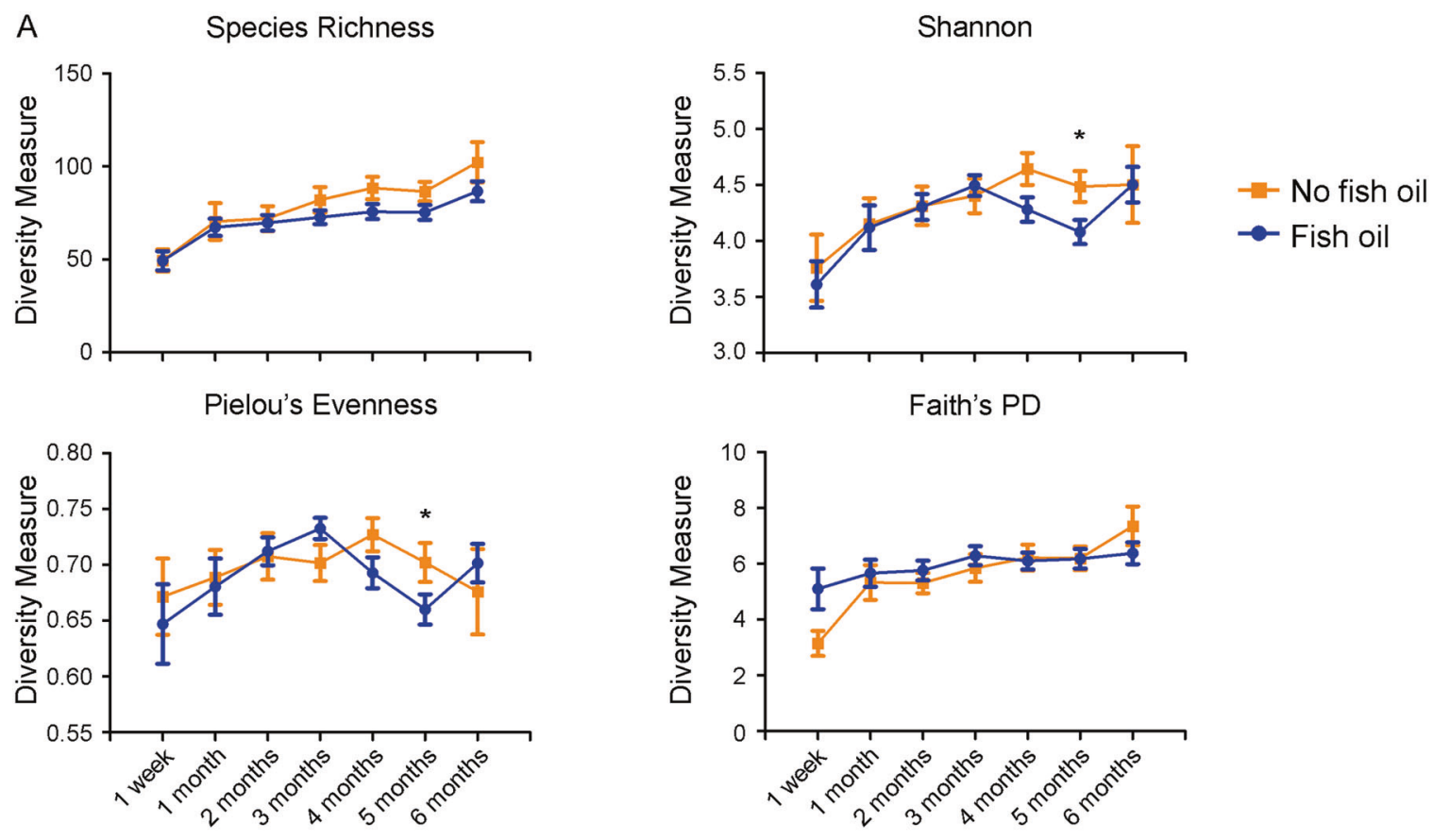

B

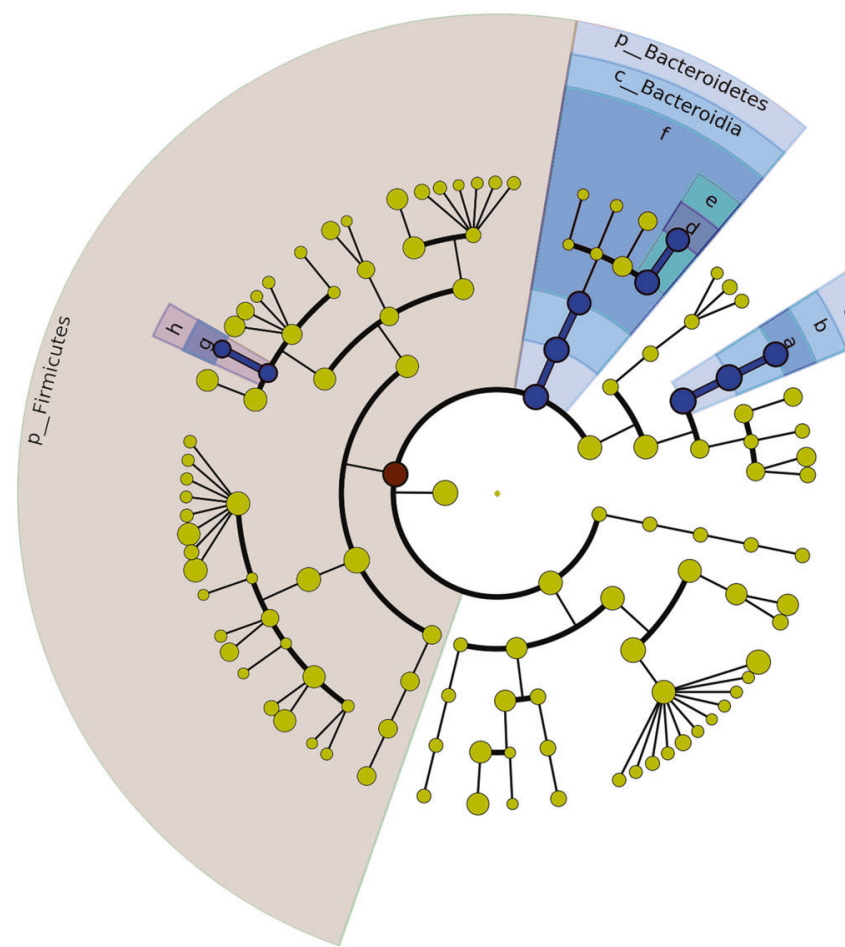

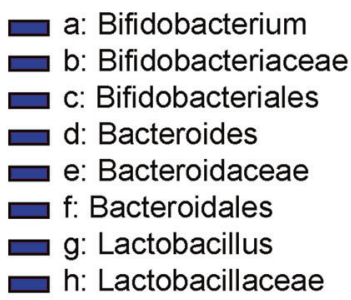

Fig. 2 Fish oil supplements taken during lactation increase antiinflammatory microbes and alter bacterial taxa succession. a Alpha diversity among supplementing groups over a 6-month period using Shannon, Faith's PD, Pielou's Evenness, and Observed Species Richness measures, $* P<0.05$. b LEfSe results comparing stool from infants in the fish oil $(n=6)$ group and no fish oil $(n=7)$ group at 1 week of age. The cladogram reports the taxa showing different abundance values according to LEfSe. Colors indicate the lineages that are encoded within corresponding samples. increase in Bifidobacterium spp. in the fish oil supplemented cohort, which had increased breast milk EPA (Fig. 4c). In order to explore the relationship between the microbial community and breast milk fatty acids for both 3- and 4-month-old infant data and to test the significance of the identified relationships, Procrustes rotation 
Table 2 Multiple regression summary of species richness, evenness, and Faith's PD data.

\begin{tabular}{|c|c|c|c|c|c|c|c|}
\hline \multirow[t]{2}{*}{ Age } & \multirow{2}{*}{$\begin{array}{l}\text { Alpha } \\
\text { diversity }\end{array}$} & \multirow[t]{2}{*}{ Variable } & \multicolumn{2}{|c|}{ Unstandardized coefficients } & \multirow{2}{*}{$\begin{array}{l}\text { Standardized } \\
\text { coefficients } \\
\text { Beta }\end{array}$} & \multirow[t]{2}{*}{$t$} & \multirow[t]{2}{*}{$P$} \\
\hline & & & $\mathrm{B}$ & Standard error & & & \\
\hline \multirow[t]{6}{*}{2 months } & \multirow[t]{3}{*}{ Richness } & C22:6n-3 & -115.22 & 44.07 & \multirow[t]{3}{*}{-0.45} & -2.615 & $0.017 *$ \\
\hline & & Sex & -5.49 & 7.15 & & -0.77 & 0.45 \\
\hline & & MOD & 18.37 & 7.75 & & 2.37 & $0.028^{*}$ \\
\hline & \multirow[t]{3}{*}{ Faith's PD } & $C 22: 6 n-3$ & -6.81 & 3.26 & \multirow[t]{3}{*}{-0.39} & -2.09 & $0.049 *$ \\
\hline & & Sex & -0.84 & 0.53 & & -1.58 & 0.13 \\
\hline & & MOD & 0.63 & 0.57 & & 1.1 & 0.29 \\
\hline \multirow[t]{6}{*}{3 months } & \multirow[t]{3}{*}{ Shannon } & C22:6n-3 & -1.62 & 0.98 & \multirow[t]{3}{*}{-0.16} & -1.67 & 0.11 \\
\hline & & Sex & -0.11 & 0.2 & & -0.57 & 0.58 \\
\hline & & MOD & 0.55 & 0.21 & & 2.6 & $0.017 *$ \\
\hline & \multirow[t]{3}{*}{ Faith's PD } & $\mathrm{C} 16: 0$ & 0.14 & 0.068 & \multirow[t]{3}{*}{0.366} & 2.09 & $0.049 *$ \\
\hline & & Sex & -1.01 & 0.43 & & -2.36 & $0.029^{*}$ \\
\hline & & MOD & 0.74 & 0.47 & & 1.6 & 0.13 \\
\hline \multirow[t]{9}{*}{4 months } & \multirow[t]{3}{*}{ Richness } & $C 22: 6 n-3$ & -195.33 & 65.83 & \multirow[t]{3}{*}{-0.49} & -2.97 & $0.008 * *$ \\
\hline & & Sex & -19.21 & 7.9 & & -2.43 & $0.025^{*}$ \\
\hline & & MOD & 14.14 & 8.67 & & 1.63 & 0.12 \\
\hline & \multirow[t]{3}{*}{ Faith's PD } & $C 22: 6 n-3$ & -12.87 & 4.33 & \multirow[t]{3}{*}{-0.51} & -2.97 & $0.008 * *$ \\
\hline & & Sex & -1.18 & 0.52 & & -2.27 & $0.035^{*}$ \\
\hline & & MOD & 0.013 & 0.57 & & 0.023 & 0.98 \\
\hline & \multirow[t]{3}{*}{ Shannon } & $C 22: 6 n-3$ & -4.55 & 1.89 & \multirow[t]{3}{*}{-0.45} & -2.4 & $0.026^{*}$ \\
\hline & & Sex & -0.39 & 0.23 & & -1.71 & 0.1 \\
\hline & & MOD & 0.19 & 0.25 & & 0.77 & 0.45 \\
\hline
\end{tabular}

Variables were selected by the model from the following lipids: C13:0, C16:0, C18:1n-9, C18:2n-6, C20:1n9, C20:5n-3, C22:5n-3, and C22:6n-3 (n=24). $P<0.05 ; * * P<0.01$. and PROTEST permutation test were applied to the RDA ordination results. The correlation diagnostics gave a Procrustes sum of squares value $\left(m_{12}\right)$ of 0.55 and a correlation in symmetric Procrustes rotation of 0.6682 which was significant $(P=0.001)$ when using 3 - and 4-month RDA scores, respectively. This suggests a high degree of correspondence between the 3- and 4-month assemblages. Overall, breast milk EPA consistently aligns with abundances of Bifidobacterium, and MOD aligns with Bacteroides at 3 and 4 months of age. Oleic acid, tridecylic acid, and DHA were also highlighted as potential predictors of microbial diversity but the results were not consistent over time.

\section{Fish oil predicts reduced commensal colonization resistance}

We furthered our exploratory analysis by assessing whether fish oil was associated with any predicted high-level phenotypes. The results revealed a predicted increase in the relative abundance of Gram-positive bacteria in the fish oil cohort and a corresponding decrease in Gram-negative bacteria at 2 months of age (Fig. 5a, b). In addition, our findings indicate that fish oil supplementation corresponded with lower abundances of biofilm forming, mobile element containing, and stress tolerant bacteria at 2 months of age (Fig. 5c-e).

\section{Fish oil supplementation does not associate with changes to rates of infections in infants}

Nineteen mothers in the fish oil cohort and ten mothers in the no fish oil cohort participated in a 2-year follow-up on their infant's health. Unlike the mothers' breast milk, infant stool did not reveal any differences in cytokines, $\operatorname{sIgA}$, or IgE expression at 1 week, 1, or 5 months of age (Table S3) and the LDA which incorporated immune markers collectively was unable to predict the supplementing group at any time point. Similarly, both incidences of mucosal infections and time required to clear mucosal infections (duration) were statistically insignificant between the groups (Fig. 5f). On average, the mothers supplemented with fish oil reported that their infant was sick $3.45 \pm 0.61$ times over a 2 -year period, with the non-supplementing group reporting $4.38 \pm 1.52$ times. The fish oil and no fish oil infants took an average of $8.07 \pm 1.12$ and $5.86 \pm 1.43$ days to clear their infections, respectively. 

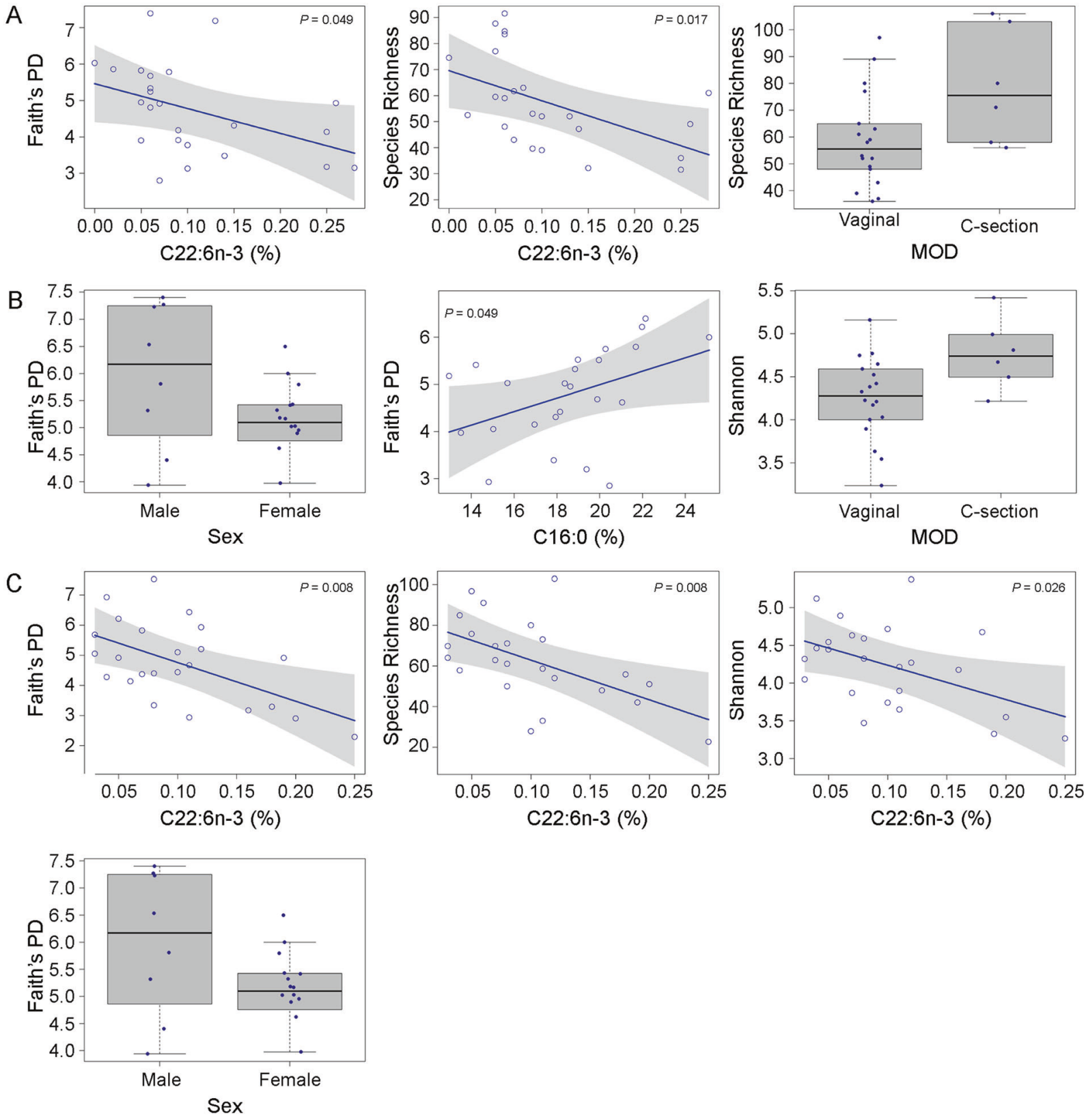

Fig. 3 Results of a multiple regression model show a significant association between alpha diversity measures and four of ten predictors of interest. a At 2 months of age, Faith's PD and Species richness negatively correlate with breast milk DHA (C22:6n-3) levels. Species richness also significantly correlates with MOD. b At 3 months, Faith's PD positively correlates with sex and palmitic acid

(C16:0). Shannon diversity significantly correlates with MOD at 3 months of age. c Correlations among species richness, Faith's PD and Shannon diversity show a negative association with DHA at 4 months. Faith's PD also correlates with sex. Shaded areas represent $95 \%$ confidence intervals $(n=24)$.

\section{Discussion}

While maternal fish oil supplementation is widely believed to support infant health, the effect on gut health is relatively unknown. This is the first study aimed at investigating the effects of habitual fish oil supplementation on breast milk fatty acid composition and the infant microbial composition from birth until 6 months of age with corresponding health

outcomes up to 2 years. Here we show that women supplementing with fish oil associate with decreased defensive inflammatory responses and produce an infant gut bacteriome with potentially less capacity to prime the proinflammatory branches of the immune system in their infants. Overall, habitual fish oil supplementation was not associated with clear and better health outcomes in supplemented infants. 
Table 3 Identified known taxa across supplementing groups.

\begin{tabular}{|c|c|c|c|c|c|c|c|c|c|}
\hline \multirow[t]{2}{*}{ Group } & \multicolumn{3}{|c|}{2 months of age } & \multicolumn{3}{|c|}{3 months of age } & \multicolumn{3}{|c|}{4 months of age } \\
\hline & Fish oil & No fish oil & Total & Fish oil & No fish oil & Total & Fish oil & No fish oil & Total \\
\hline Phylum & 9 & 9 & 9 & 9 & 9 & 9 & 9 & 9 & 9 \\
\hline Class & 15 & 17 & 17 & 15 & 16 & 17 & 15 & 16 & 17 \\
\hline Order & 22 & 24 & 27 & 22 & 25 & 27 & 24 & 24 & 27 \\
\hline Family & 36 & 36 & 47 & 36 & 42 & 49 & 41 & 38 & 47 \\
\hline Genus & 55 & 56 & 83 & 54 & 57 & 84 & 64 & 61 & 84 \\
\hline
\end{tabular}

Summary of the number of taxa across all participants as categorized based on their age and fish oil supplementation $(n=24)$.
Fish oil has two major n-3 PUFAs important for infant development: EPA and DHA. A recent systematic review [53] showed that with one exception [54], controlled studies consistently supplement mothers with higher DHA than EPA, and often with DHA exclusively. However, in this study it was apparent that Canadian mothers supplement with higher EPA than DHA, resulting in relatively higher proportions of EPA in breast milk. In this study, we show habitual administration of fish oil correlated with a greater proportion of breast milk EPA, accompanied by a marked decrease in the proportion of AA throughout lactation when compared with the non-supplementing participants, similar to previous studies [55, 56]. Fish oil supplementation also resulted in less eicosatrienoic acid, a less reported n-3 PUFA. While eicosatrienoic acid has widely been considered a dead-end product, recent studies show desaturation of eicosatrienoic acid yields eicosatetraenoic acid (C20:4n-3) as well as immediate eicosanoid precursors [57], which is consistent with the idea that fish oil decreases inflammatory mediators [58].

This study shows that fish oil supplementation, primarily driven by EPA, decreases defensive inflammation in breast milk. Breast milk supplies the first source of antigenspecific immune protection in the intestinal tract of infants in the form of $\operatorname{sgA}$. sIgA has been shown to shape the composition of the gut microbiome and prevent the translocation of aerobic bacteria from the neonatal gut into lymph nodes [59]. This protects against inflammation and promotes intestinal barrier function. This study found that breast milk sIgA was decreased in the supplementing mother's breast milk at 5 months postpartum and this corresponded with increased levels of EPA. This is similar to previous studies showing decreased breast milk concentrations of sIgA corresponding with increased breast milk n-3 PUFA through fish consumption [60]. In addition, the antiinflammatory cytokine IL-10 was increased in mother's breast milk who supplemented with fish oil. IL-10 inhibits the activity of pro-inflammatory Th1 cells, NK cells, and macrophages, leading to impaired pathogen control and clearance which can result in persistent asymptomatic infections including Candida albicans and mycobacterial infections (reviewed in [61]). Overall, fish oil supplementation decreased critically important defense factors in one of the only sources the infants get during early life.

In addition to reducing breast milk defense factors, there were limited differences in the infant gut bacteriome. Fish oil supplementation was associated with higher Bifidobacteria and Lactobacillus spp. at 1 week of age with no overall differences in fecal alpha diversity. To understand which specific breast milk fatty acids are good predictors of microbial diversity, complete data sets at months 2,3 , and 4 were reassessed according to mothers' breast milk fatty acid profile. Results from a regression model showed that higher levels of DHA in breast milk negatively correspond with fecal species richness and phylogenetic diversity at 2 and 4 months of age. DHA proportions in breast milk were likewise shown to contribute to microbial assemblages in infant stool at 3 and 4 months of age. Although none of the selected breast milk fatty acids correlated with bacterial abundance at 2 months, our Procrustes results suggest a high degree of concordance between the environmental variables EPA and DHA on microbial assemblages at 3 and 4 months. One notable finding of this study is that n-3 PUFAs illicit a stronger effect on the infant bacteriome than other more abundant breast milk fatty acids. In fact, the global RDA model selected both EPA and DHA as meaningful explanatory variables alongside MOD, which is a known significant contributor to microbial diversity. Similar to the results seen in the fish oil group at 1 week, the RDA results investigating the association between individual fatty acids and the infant gut further showed that EPA in breast milk uniquely posed a significant association with members of Bifidobacterium, Lactobacillus, and Streptococcus, independent of DHA. This was verified by LEfSe that showed an increase in Bifidobacterium spp. in the fish oil supplementing subset at 3 and 4 months of age. In support of previous literature, our study shows that Bacteroides spp. were associated with infant MOD [62] and that caesarian sectioned babies have higher alpha diversity in early life [63]. We also found that female infants had lower alpha diversity at 3 and 4 months of age. These findings strongly mimic rodent models performed in our lab, which showed 
A
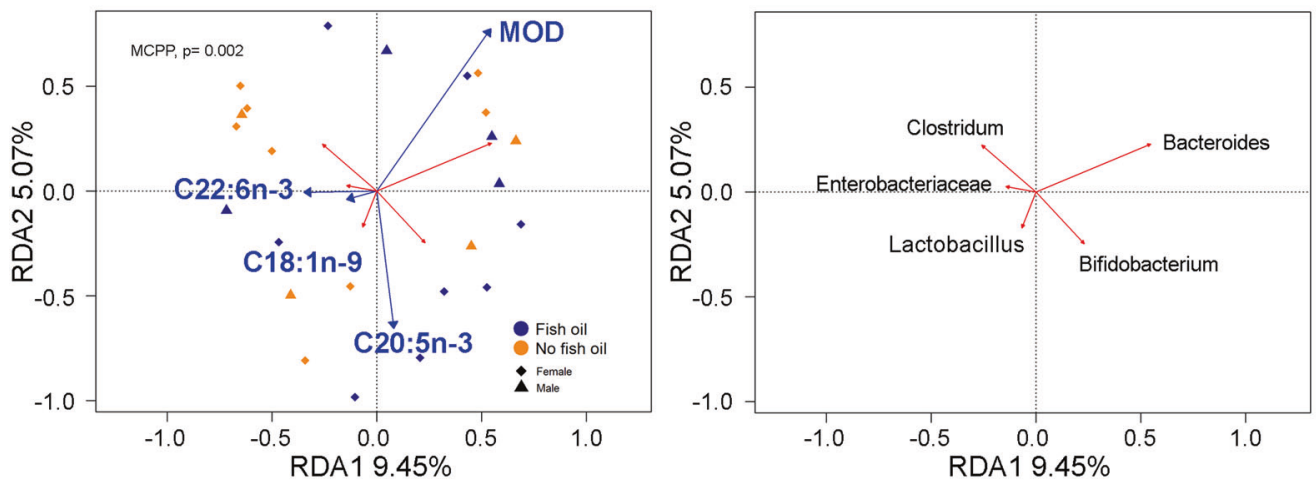

в
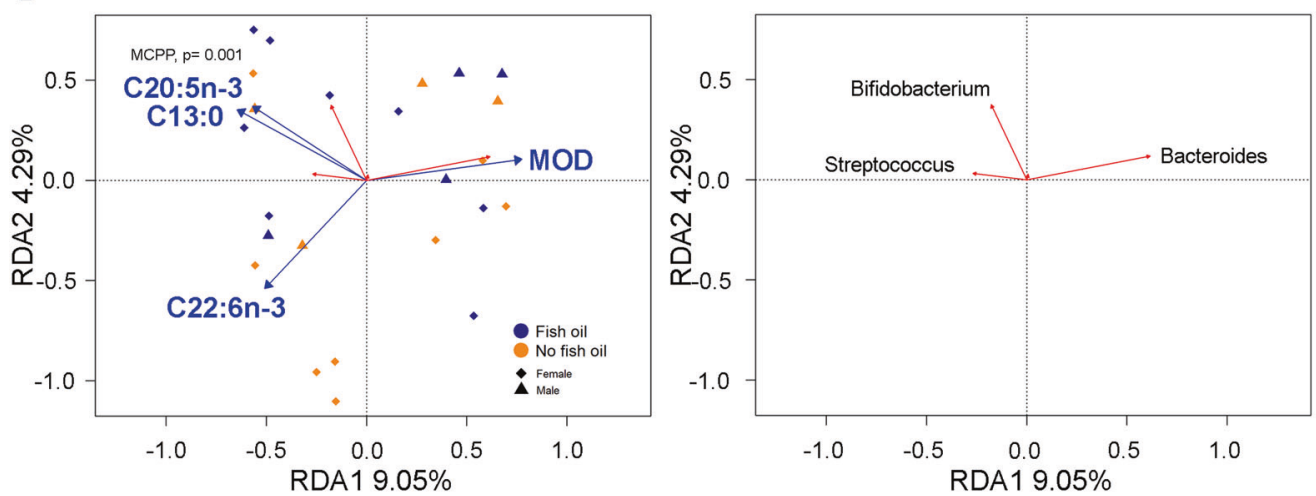

C
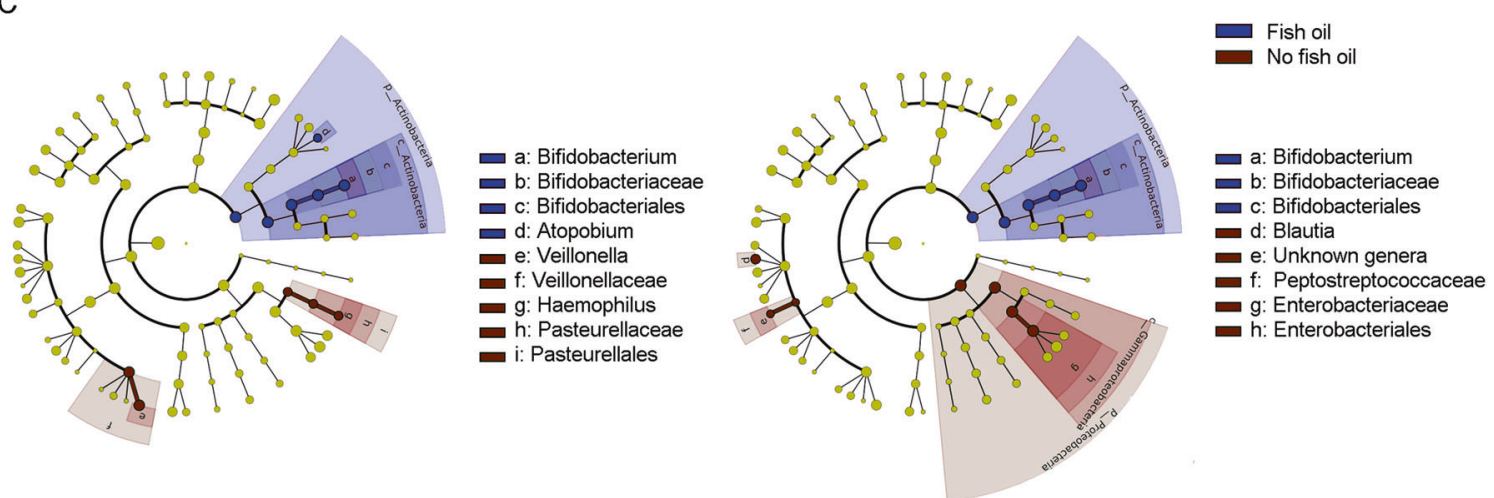

Fig. 4 Breast milk EPA (C20:5n-3) associates with antiinflammatory microbes such as Bifidobacteria and Lactobacillus spp., whereas DHA (C22:6n-3) aligns with Enterobacteriaceae. Bacterial abundance RDA correlation biplots constrained by selected explanatory variables at a 3 months of age and b 4 months of age. The groups and explanatory variables and genera plots are presented separately for clarity; however, they are derived from the same RDA model. RDA1 and RDA2 which explain over $14.52 \%$ and $13.34 \%$ of total variation in beta diversity, respectively, are plotted. The global model's $P$ value was calculated using the Monte Carlo Permutation

n-3 PUFA supplementation increases gut microbes such as Lactobacillus and Bifidobacteria spp. [17].

Previous studies have shown that Lactobacillus and Bifidobacteria spp. have the ability to induce anti-

Procedure (MCPP). Subjects are color coded according to whether or not the mother supplemented with Fish oil for illustrative purposes only as groupings were not included in the model. $P \leq 0.05$ is considered significant $(n=24)$. c LEfSe results comparing stool from infants in the fish oil $(n=24)$ group and no fish oil $(n=24)$ groups at 3 (left) and 4 (right) months of age show an increased abundance of anti-inflammatory bacteria such as Bifidobacteria spp. in the fish oil cohort. The cladogram reports the taxa showing different abundance values according to LEfSe. Colors indicate the lineages that are encoded within corresponding samples.

inflammatory response in their host [51, 64]. Generally, these species are considered beneficial; however, they proved unfavorable during enteric infection which resulted in murine sepsis and mortality [17]. In this study, we did not 

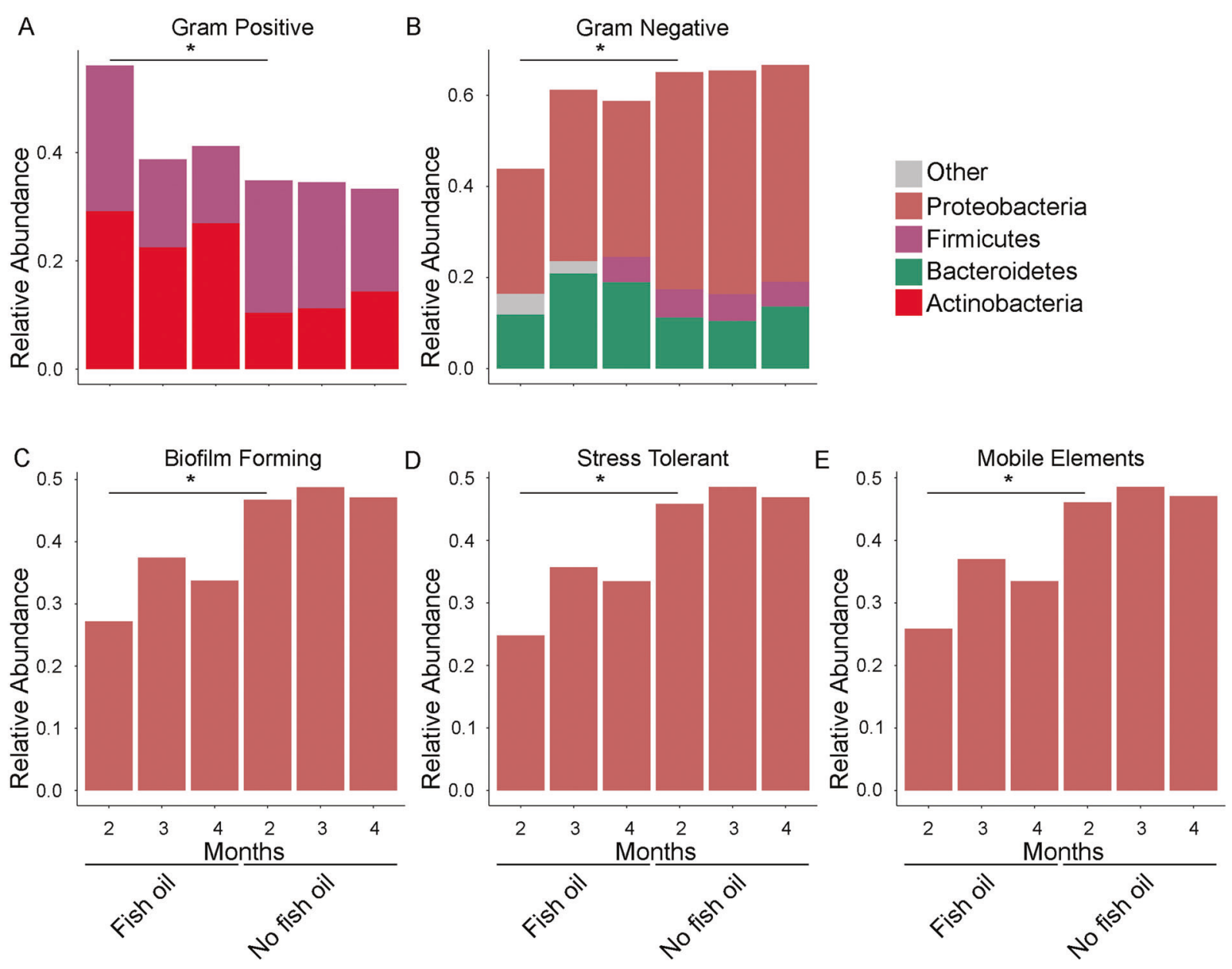

$\mathrm{F}$

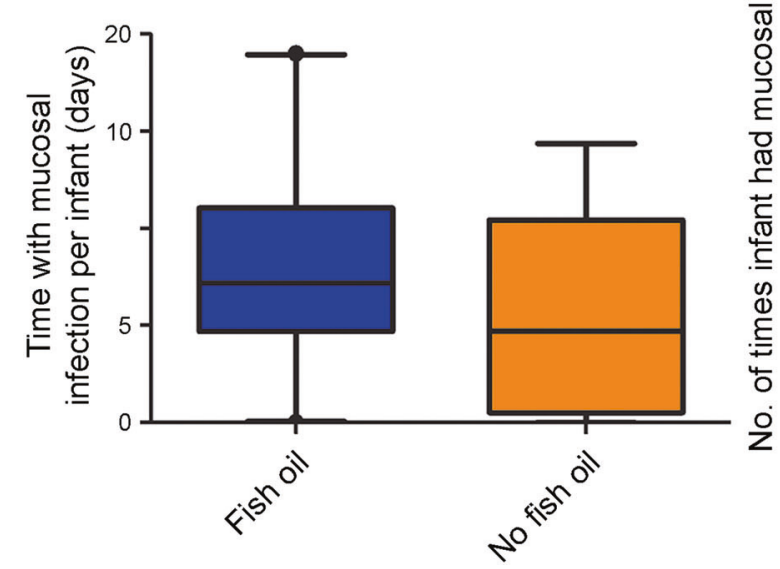

Fig. 5 Fish oil supplementation during lactation increases the predicted relative abundance of commensal virulence traits involved in pathogen colonization resistance but does not associate with rates of infections in infants. BugBase predicted relative abundance of a Gram-positive bacteria, b Gram-negative bacteria,

see any evidence that infants in the fish oil cohort had altered immune function; however, predicted microbial phenotypes suggested an inverse relationship between fish oil and biofilm formation, mobile elements and stress

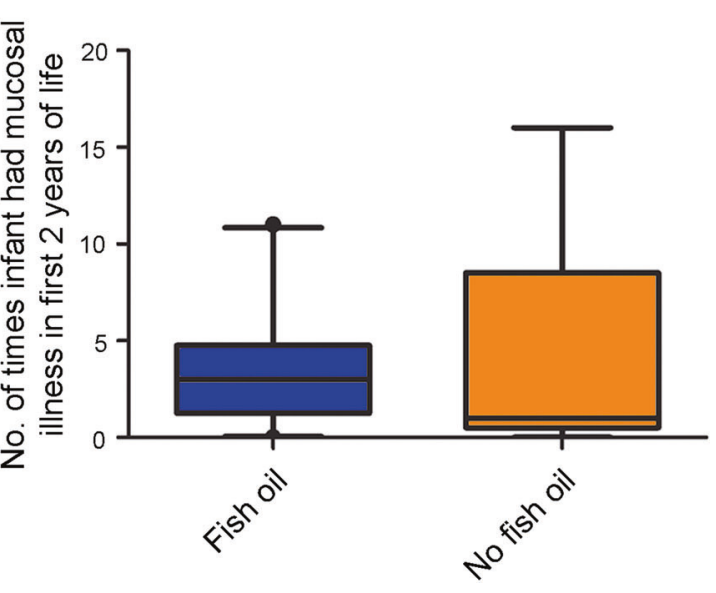

$\mathbf{c}$ biofilms formers, $\mathbf{d}$ stress tolerant bacteria, and e bacteria containing mobile elements at 2, 3 and 4 months of age. Values are expressed as mean values ( $n=24$ per group). An asterisk indicates $P<0.05$ using a pairwise Mann-Whitney-Wilcoxon test. f Infection rates and duration were not different between the two cohorts.

tolerance. While these virulence traits are usually associated with bacterial pathogens [65], it is plausible that commensal bacteria with these predictive traits may benefit the infant given the immune system is primed and developed during 
this period of time. For example, since commensal biofilms occur in close juxtaposition to host epithelium, it could indicate that these bacteria interact to a greater extent with the immune system than their planktonic counterparts and are more resistant against pathogen colonization [66]. In support of this, the gut epithelia contacted by commensal bacteria generate reactive oxygen species which could account for increased stress tolerant bacteria in this group. Microbial-elucidated reactive oxygen species can increase cellular proliferation in the intestine and modulate innate immune signaling [67]. Germ-free models have shown that in the absence of microbial exposures, the immune system is immature (reviewed here [68]) and that some, but not all, bacteria act to "mature" the immune system. For instance, previous studies have shown that Bifidobacterium longum was not able to induce the production of RegIII $\gamma$ by Paneth cells, whereas Bacteroides thetaiotaomicron was [69, 70]. Thus, the predicted decrease in biofilm formation, mobile elements, and stress tolerant bacteria in the fish oil cohort could contribute to decreased immune priming. Despite this, we did not observe any differences in infant susceptible to infection. A possible explanation for this might be that none of the children in this study were critically ill or exposed to bacterial pathogens. The "anti-inflammatory" nature of n-3 PUFA may only be detrimental when exposed to infectious pathogens.

Overall, our findings underscore that different types of breast milk fatty acids impart varying effects on the human infant bacteriome. While this was an observational prospective study, we demonstrated that supplementing with fish oil increased the percent composition of EPA in breast milk, which was associated with decreased defensive inflammatory mediators in the infant's nutrition source and this was associated with a gut microbiome that had reduced immune priming capability and predicted less colonization resistance. Given that fish oil appears to alter the infant microbial composition through changes in breast milk, and that the microbiota is intricately linked to infant health, we recommend large scale epidemiological studies to clarify whether early fish oil exposures alter infectious disease susceptibility including persistent asymptomatic chronic infections.

\section{Data availability}

The data set supporting the results of this article is available in on the Open Science Framework data depository at https://osf.io/d9x4p/.

Acknowledgements We thank Interior Health, D. Erland (RN), and Dr M. Docherty (MD) for recruitment assistance, A. Plessis for data entry, and the Okanagan community for promoting and participating in the study.

Funding This study is supported by CAG, CIHR, CCC, and NSERC.
Author contributions Conceived, designed, and funded the experiments: DLG. Managed recruitment and sample storage: DMV and MNJ. Assisted in recruitment and sample collection: MNJ, BWB, and JAB. Performed initial sIgA analysis: BWB. Performed dietary analysis: NH. Prepared all stool samples: JAB. Supervised and analyzed all GC analysis and data: SG. Assisted with microbial analysis: ME and JP. Performed the experiments and analyzed the data: CQ. Wrote the paper: CQ, DLG, with edits from JP and SG.

\section{Compliance with ethical standards}

Conflict of interest The remaining authors have declared that no competing interests exist. CQ was funded through a Canadian Association of Gastroenterology (CAG) and Canadian Institute for Health Research (CIHR) PhD awards Doctoral Research Award. DMV was funded through a Natural Sciences and Engineering Research Council (NSERC) Undergraduate Student Research Award. ME was funded through a NSERC PhD Award. JP was funded though the NSERC Discovery Grants (DG) program. SG was funded through the Canadian Diabetes Association and an NSERC DG. DLG was funded through an NSERC DG and Crohn's and Colitis Canada.

Publisher's note Springer Nature remains neutral with regard to jurisdictional claims in published maps and institutional affiliations.

\section{References}

1. Neuringer M, Connor WE, Van Petten C, Barstad L. Dietary omega-3 fatty acid deficiency and visual loss in infant rhesus monkeys. J Clin Investig. 1984;73:272-6.

2. Krabbendam L, Bakker E, Hornstra G, van Os J. Relationship between DHA status at birth and child problem behaviour at 7 years of age. Prostaglandins leukot Essent Fatty Acids. 2007;76:29-34.

3. Catalan J, Moriguchi T, Slotnick B, Murthy M, Greiner RS, Salem $\mathrm{N}$ Jr. Cognitive deficits in docosahexaenoic acid-deficient rats. Behav Neurosci. 2002;116:1022-31.

4. De Giuseppe R, Roggi C, Cena H. n-3 LC-PUFA supplementation: effects on infant and maternal outcomes. Eur $\mathrm{J}$ Nutr. 2014;53:1147-54.

5. Ghosh S, Dai C, Brown K, Rajendiran E, Makarenko S, Baker J, et al. Colonic microbiota alters host susceptibility to infectious colitis by modulating inflammation, redox status, and ion transporter gene expression. Am J Physiol Gastrointest Liver Physiol. 2011;301:G39-49.

6. Oddy WH. Breastfeeding, childhood asthma, and allergic disease. Ann Nutr Metab. 2017;70 Suppl 2 :26-36.

7. APA. Omega-3 fish oil and pregnancy. American Pregnancy Association; 2019 https://americanpregnancy.org/pregnancy-hea 1th/omega-3-fish-oil/.

8. Delgado-Noguera MF, Calvache JA, Bonfill Cosp X, Kotanidou EP, Galli-Tsinopoulou A. Supplementation with long chain polyunsaturated fatty acids (LCPUFA) to breastfeeding mothers for improving child growth and development. Cochrane Database Syst Rev. 2015;7:CD007901.

9. Simmer K, Patole SK, Rao SC. Long-chain polyunsaturated fatty acid supplementation in infants born at term. Cochrane Database Syst Rev. 2011;3:CD000376.

10. Quin C, Erland BM, Loeppky JL, Gibson DL. Omega-3 polyunsaturated fatty acid supplementation during the pre and postnatal period: a meta-analysis and systematic reivew of randomized and semi-randomized controlled trials. NJIM. 2016;5:34-54.

11. Das UN. Essential fatty acids and their metabolites could function as endogenous HMG-CoA reductase and ACE enzyme inhibitors, 
anti-arrhythmic, anti-hypertensive, anti-atherosclerotic, antiinflammatory, cytoprotective, and cardioprotective molecules. Lipids Health Dis. 2008;7:37.

12. Rose DP, Connolly JM. Omega-3 fatty acids as cancer chemopreventive agents. Pharm Ther. 1999;83:217-44.

13. James MJ, Gibson RA, Cleland LG. Dietary polyunsaturated fatty acids and inflammatory mediator production. Am J Clin Nutr. 2000;71 1 Suppl 343S-8S.

14. Calder PC. N-3 polyunsaturated fatty acids and inflammation: from molecular biology to the clinic. Lipids. 2003;38:343-52.

15. Chambers CA, Allison JP. Costimulatory regulation of $\mathrm{T}$ cell function. Curr Opin Cell Biol. 1999;11:203-10.

16. Fenton JI, Hord NG, Ghosh S, Gurzell EA. Immunomodulation by dietary long chain omega- 3 fatty acids and the potential for adverse health outcomes. Prostaglandins leukot Essent Fatty Acids. 2013;89:379-90.

17. Ghosh S, DeCoffe D, Brown K, Rajendiran E, Estaki M, Dai C, et al. Fish oil attenuates omega- 6 polyunsaturated fatty acidinduced dysbiosis and infectious colitis but impairs LPS dephosphorylation activity causing sepsis. PloS ONE. 2013;8:e55468.

18. DeCoffe D, Quin C, Gill SK, Tasnim N, Brown K, Godovannyi A, et al. Dietary lipid type, rather than total number of calories, alters outcomes of enteric infection in mice. J Infect Dis. 2016;213:1846-56.

19. Woodworth HL, McCaskey SJ, Duriancik DM, Clinthorne JF, Langohr IM, Gardner EM, et al. Dietary fish oil alters T lymphocyte cell populations and exacerbates disease in a mouse model of inflammatory colitis. Cancer Res. 2010;70:7960-9.

20. McFarland CT, Fan YY, Chapkin RS, Weeks BR, McMurray DN. Dietary polyunsaturated fatty acids modulate resistance to Mycobacterium tuberculosis in guinea pigs. $J$ Nutr. 2008;138:2123-8.

21. Bonilla DL, Fan YY, Chapkin RS, McMurray DN. Transgenic mice enriched in omega-3 fatty acids are more susceptible to pulmonary tuberculosis: impaired resistance to tuberculosis in fat1 mice. J Infect Dis. 2010;201:399-408.

22. Irons R, Anderson MJ, Zhang M, Fritsche KL. Dietary fish oil impairs primary host resistance against Listeria monocytogenes more than the immunological memory response. J Nutr. 2003;133:1163-9.

23. Cruz-Chamorro L, Puertollano MA, Puertollano E, Alvarez de Cienfuegos G, de Pablo MA. Examination of host immune resistance against Listeria monocytogenes infection in cyclophosphamide-treated mice after dietary lipid administration. Clin Nutr. 2007;26:631-9.

24. Snel J, Born L, van der Meer R. Dietary fish oil impairs induction of gamma-interferon and delayed-type hypersensitivity during a systemic Salmonella enteritidis infection in rats. APMIS. 2010;118:578-84.

25. Oarada M, Tsuduki T, Suzuki T, Miyazawa T, Nikawa T, Hongquan G, et al. Dietary supplementation with docosahexaenoic acid, but not with eicosapentaenoic acid, reduces host resistance to fungal infection in mice. Biochim Biophys Acta. 2003;1622:151-60.

26. Byleveld PM, Pang GT, Clancy RL, Roberts DC. Fish oil feeding delays influenza virus clearance and impairs production of interferon-gamma and virus-specific immunoglobulin $\mathrm{A}$ in the lungs of mice. J Nutr. 1999;129:328-35.

27. Byleveld M, Pang GT, Clancy RL, Roberts DC. Fish oil feeding enhances lymphocyte proliferation but impairs virus-specific $\mathrm{T}$ lymphocyte cytotoxicity in mice following challenge with influenza virus. Clin Exp Immunol. 2000;119:287-92.

28. Schwerbrock NM, Karlsson EA, Shi Q, Sheridan PA, Beck MA. Fish oil-fed mice have impaired resistance to influenza infection. J Nutr. 2009;139:1588-94.
29. Gibson D, Gill S, Brown K, Tasnim N, Ghosh S, Innis S, et al. Maternal exposure to fish oil primes offspring to harbor intestinal pathobionts associated with altered immune cell balance. Gut Microbes. 2015;6:24-32.

30. Innis SM, Dai C, Wu X, Buchan AM, Jacobson K. Perinatal lipid nutrition alters early intestinal development and programs the response to experimental colitis in young adult rats. Am J Physiol Gastrointest Liver Physiol. 2010;299:G1376-85.

31. Quin C, Estaki M, Vollman DM, Barnett JA, Gill SK, Gibson DL. Probiotic supplementation and associated infant gut microbiome and health: a cautionary retrospective clinical comparison. Sci Rep. 2018;8:8283.

32. Gorzelak MA, Gill SK, Tasnim N, Ahmadi-Vand Z, Jay M, Gibson DL. Methods for improving human gut microbiome data by reducing variability through sample processing and storage of stool. PLoS ONE. 2015;10:e0134802.

33. Kang JX, Wang J. A simplified method for analysis of polyunsaturated fatty acids. BMC Biochem. 2005;6:5.

34. Jovel J, Patterson J, Wang W, Hotte N, O'Keefe S, Mitchel T, et al. Characterization of the gut microbiome using $16 \mathrm{~S}$ or shotgun metagenomics. Front Microbiol. 2016;7:459.

35. Bolyen ER, Dillon JR, Bokulich MR, Abnet NA, Al-Ghalith C, Alexander GA, et al. QIIME 2: reproducible, interactive, scalable, and extensible microbiome data science. PeerJ Prepr. 2018;6: e27295v2.

36. Amir A, McDonald D, Navas-Molina JA, Kopylova E, Morton JT, Zech Xu Z, et al. Deblur rapidly resolves single-nucleotide community sequence patterns. mSystems. 2017;2:e0191-16.

37. Katoh K, Standley DM. MAFFT multiple sequence alignment software version 7: improvements in performance and usability. Mol Biol Evol. 2013;30:772-80.

38. Price MN, Dehal PS, Arkin AP. FastTree 2-approximately maximum-likelihood trees for large alignments. PloS ONE 2010;5: e9490.

39. R Core Team. R: a language and environment for statistical computing. Vienna: R Foundation for Statistical Computing; 2014.

40. Segata N, Izard J, Waldron L, Gevers D, Miropolsky L, Garrett WS, et al. Metagenomic biomarker discovery and explanation. Genome Biol. 2011;12:R60.

41. Ward T, Larson J, Meulemanns J, Hillmann B, Lynch J, Sidiropoulos D, et al. BugBase predicts organism level microbiome phenotypes. 2017 https://doi.org/10.1101/133462.

42. Rognes T, Flouri T, Nichols B, Quince C, Mahe F. VSEARCH: a versatile open source tool for metagenomics. PeerJ. 2016;4:e2584.

43. Estaki M, Pither J, Baumeister P, Little JP, Gill SK, Ghosh S, et al. Cardiorespiratory fitness as a predictor of intestinal microbial diversity and distinct metagenomic functions. Microbiome. 2016;4:42.

44. Legendre P, Gallagher ED. Ecologically meaningful transformations for ordination of species data. Oecologia. 2001;129:271-80.

45. Wang Y, Naumann U, Eddelbuettel D, Wilshire J, Warton D, Byrnes J, et al. mvabund: statistical methods for analysing multivariate abundance data. $\mathrm{R}$ package version 3.12 .3 ; 2016. https:// cran.r-project.org/web/packages/mvabund/mvabund.pdf.

46. Nielsen S, Needham B, Leach ST, Day AS, Jaffe A, Thomas T, et al. Disrupted progression of the intestinal microbiota with age in children with cystic fibrosis. Sci Rep. 2016;6:24857.

47. Peres-Neto PR, Jackson DA. How well do multivariate data sets match? The advantages of a Procrustean superimposition approach over the Mantel test. Oecologia. 2001;129:169-78.

48. Mardia KV, Kent JT, Bibby JM. Multivariate analysis. Academic Press; 1979.

49. Venables WN, Ripley BD. Statistics and computing: modern applied statistics with S. Springer; New York: Springer-Verlag; 2002. https://doi.org/10.1007/978-0-387-21706-2. 
50. Yazdanbakhsh M, Kremsner PG, van Ree R. Allergy, parasites, and the hygiene hypothesis. Science. 2002;296:490-4.

51. Oh NS, Joung JY, Lee JY, Kim Y. Probiotic and antiinflammatory potential of Lactobacillus rhamnosus 4B15 and Lactobacillus gasseri 4M13 isolated from infant feces. PLoS ONE. 2018;13:e0192021.

52. Okada Y, Tsuzuki Y, Hokari R, Komoto S, Kurihara C, Kawaguchi A, et al. Anti-inflammatory effects of the genus Bifidobacterium on macrophages by modification of phospho-I kappaB and SOCS gene expression. Int J Exp Pathol. 2009;90:131-40.

53. Amaral YN, Marano D, Silva LM, Guimaraes AC, Moreira ME. Are there changes in the fatty acid profile of breast milk with supplementation of omega-3 sources? A systematic review. Rev Bras Ginecol Obstet. 2017;39:128-41.

54. Boris J, Jensen B, Salvig JD, Secher NJ, Olsen SF. A randomized controlled trial of the effect of fish oil supplementation in late pregnancy and early lactation on the n-3 fatty acid content in human breast milk. Lipids. 2004;39:1191-6.

55. Dunstan JA, Roper J, Mitoulas L, Hartmann PE, Simmer K, Prescott SL. The effect of supplementation with fish oil during pregnancy on breast milk immunoglobulin A, soluble CD14, cytokine levels and fatty acid composition. Clin Exp Allergy. 2004;34:1237-42.

56. Helland IB, Saugstad OD, Saarem K, Van Houwelingen AC, Nylander G, Drevon CA. Supplementation of n-3 fatty acids during pregnancy and lactation reduces maternal plasma lipid levels and provides DHA to the infants. J Matern Fetal Neonatal Med. 2006;19:397-406.

57. Park WJ, Kothapalli KS, Lawrence P, Tyburczy C, Brenna JT. An alternate pathway to long-chain polyunsaturates: the FADS2 gene product Delta8-desaturates 20:2n-6 and 20:3n-3. J Lipid Res. 2009;50:1195-202.

58. Calder PC. Omega-3 polyunsaturated fatty acids and inflammatory processes: nutrition or pharmacology? $\mathrm{Br} \mathrm{J}$ Clin Pharm. 2013;75:645-62.

59. Rogier EW, Frantz AL, Bruno ME, Wedlund L, Cohen DA, Stromberg AJ, et al. Secretory antibodies in breast milk promote long-term intestinal homeostasis by regulating the gut microbiota and host gene expression. Proc Natl Acad Sci USA. 2014; 111:3074-9.

60. Urwin HJ, Miles EA, Noakes PS, Kremmyda LS, Vlachava M, Diaper ND, et al. Salmon consumption during pregnancy alters fatty acid composition and secretory $\operatorname{Ig}$ A concentration in human breast milk. J Nutr. 2012;142:1603-10.

61. Couper KN, Blount DG, Riley EM. IL-10: the master regulator of immunity to infection. J Immunol. 2008;180:5771-7.

62. Dominguez-Bello MG, De Jesus-Laboy KM, Shen N, Cox LM, Amir A, Gonzalez A, et al. Partial restoration of the microbiota of cesarean-born infants via vaginal microbial transfer. Nat Med. 2016;22:250-3.

63. Bokulich NA, Chung J, Battaglia T, Henderson N, Jay M, Li H, et al. Antibiotics, birth mode, and diet shape microbiome maturation during early life. Sci Transl Med. 2016;8:343ra82.

64. Riedel CU, Foata F, Philippe D, Adolfsson O, Eikmanns BJ, Blum S. Anti-inflammatory effects of bifidobacteria by inhibition of LPS-induced NF-kappaB activation. World J Gastroenterol. 2006;12:3729-35.

65. Schroeder M, Brooks BD, Brooks AE. The complex relationship between virulence and antibiotic resistance. Genes. 2017;8:39.

66. Da ReS, Valle J, Charbonnel N, Beloin C, Latour-Lambert P, Faure $\mathrm{P}$, et al. Identification of commensal Escherichia coli genes involved in biofilm resistance to pathogen colonization. PloS ONE. 2013;8:e61628.

67. Jones RM, Mercante JW, Neish AS. Reactive oxygen production induced by the gut microbiota: pharmacotherapeutic implications. Curr Med Chem. 2012;19:1519-29.

68. Round JL, Mazmanian SK. The gut microbiota shapes intestinal immune responses during health and disease. Nat Rev Immunol. 2009;9:313-23.

69. Cash HL, Whitham CV, Behrendt CL, Hooper LV. Symbiotic bacteria direct expression of an intestinal bactericidal lectin. Science. 2006;313:1126-30.

70. Sonnenburg JL, Chen CT, Gordon JI. Genomic and metabolic studies of the impact of probiotics on a model gut symbiont and host. PLoS Biol. 2006;4:e413. 